\title{
Mobile Data Services using Open Protocols and Internet
}

\author{
Nitin Gupta \\ College of Engineering Roorkee \\ Roorkee, India
}

\author{
Rahul Sharma \\ IISC \\ Bangalore, India
}

\begin{abstract}
The aim of this work is to develop such a methodology whose implementation help the companies, institutions etc to easily communicate with another person without any cost. This gives a new dimension to communication. Our system is competent without GPRS or Telecom service providers. Free of cost communication with the help of this system along with some enhanced security features which will provide a hassle free and safe communication. Person often wants to share mobile data like images, videos, etc. to our distant friends. This technology removes the overhead of copying data to PC/Laptop then sends over the internet and reverse process on the other hand. Whether the person is in car, train or anywhere else and he/she has Bluetooth enabled mobile phone and Laptop then the person can transfer any urgent data to any person who also fulfills such requirements. To accomplish our task we have successfully implemented the communication, using messages and voice mails, and media/data file transfer.
\end{abstract}

Our design is based on request-reply architecture. We have also implemented an access control mechanism that allows user to receive services from everyone except for the members of the blacklist. This is necessary as the service involves virtually no cost. To authenticate the sender currently we are using a PIN number because to detect the IMEI number of the cell phone our mobile application must be digitally signed by the mobile vendor.

\section{General Terms}

Computer Communication Network (Data communication), Distributed Systems (Client/server), Design and Reliability

\section{Keywords}

Bluetooth, Wireless, Java, Mobile, Technology

\section{INTRODUCTION}

Today, for all companies, institutions and home, internet is of the essence. Internet is becoming the key to explore the various fields of information industry through its imaginative uses. The power of internet lies in its availability, wider reach, cost and speed. Internet, laptop/PC and mobile become a part of our life. Then why one has to waste money in sending messages, audio/video/data files and voice mails?
Wireless technologies are becoming more and more popular around the world. Consumers appreciate the wireless lifestyle, relieving them of the well known "cable chaos" that tends torelieving them of the well known "cable chaos" that tends to grow under their desk. Nowadays, the world would virtually stop if wireless communications suddenly became unavailable. Both our way of life and the global economy are highly dependent on the flow of information through wireless mediums like television and radio. Cell phones have become highly available during the last decade. Now virtually everyone owns a cell phone, making people available almost wherever they are. Many companies are highly dependent on their employees having cell phones, some companies have even decided not to employ stationary phone systems but instead use cell phones exclusively throughout the organization. New wireless technologies are introduced at an increasing rate. During the last few years the IEEE 802.11 [1] technologies have started to spread rapidly, enabling consumers to set up their own wireless networks. This constitutes an important change in how wireless communications are made available to consumers. As an increasing number of useful Bluetooth applications become available, many consumers will already have Bluetooth devices and be ready to start using Bluetooth PANs (Personal Area Networks) where all their Bluetooth devices communicate with one another [2].

The Java APIs for Bluetooth Wireless Technology (JABWT) ties the Java technology and the Bluetooth technology together. At the time of writing there are only two books discussing JABWT [3], [4]. These books provide quite complex code samples for Java Bluetooth programming, making it hard to grasp how JABWT is used. In this work our aim is to develop a free call transfer using Bluetooth and laptops. But due to some restrictions by nokia vendors, we have to change our idea to voice mail transfer. Now our software is capable of transferring a Voice Mail, Data Files(any format), Video(any format), Audio(any format), Message, Blacklist using a Bluetooth.

\section{BLUETOOTH}

Bluetooth is a low cost, low power; short-range radio technology intended to replace cable connections between cell phones, PDAs and other portable devices. It can clean up your desk considerably, making wires between your workstation, mouse, laptop computer etc. obsolete. Ericsson Mobile Communications started developing the Bluetooth system in 1994, looking for a replacement to the cables connecting cell phones and their accessories. The Bluetooth system is named after a tenth-century 
Danish Viking king, Harald Blatant, who united and controlled Norway and Denmark. The first Bluetooth devices hit the market around 1999. The layers of the Bluetooth stack (shown in figure 1) in the Bluetooth book by Bray and Sturman [5] and in the Bluetooth specification [6]. The Bluetooth security white paper [7] defines a security architecture which may be used to implement security mode 2 service level enforced security.

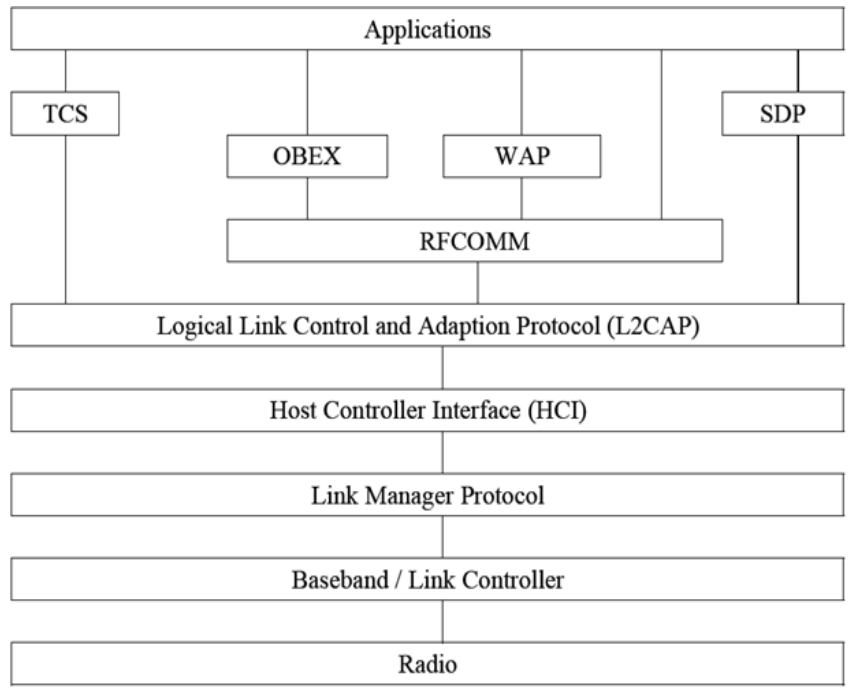

Figure 1: Bluetooth Protocol Stack

\section{BLUECOVE}

BlueCove is a Java library for Bluetooth (JSR-82 implementation) that currently interfaces with the Mac OS X, WIDCOMM, BlueSoleil and Microsoft Bluetooth stack found in Windows XP SP2 or Windows Vista and WIDCOMM and Microsoft Bluetooth stack on Windows Mobile. BlueCove-GPL is additional GPL licensed module to support BlueCove runtime on Linux BlueZ. BlueCove JSR-82 Emulator module is additional module for BlueCove to simulate Bluetooth stack. BlueCove can be used in Java Standard Edition (J2SE) 1.1 or newer. BlueCove provides JSR-82 Java interface for following Bluetooth Profiles:

SDAP - Service Discovery Application Profile RFCOMM - Serial Cable Emulation Protocol L2CAP - Logical Link Control and Adaptation Protocol OBEX - Generic Object Exchange Profile (GOEP) profile on top of RFCOMM

\section{JSR-8}

JSR-82 is a standard defined by the Java Community Process for providing a standard to develop Bluetooth applications in Java. It is an open and non-proprietary standard for developing Bluetooth applications. The JSR-82 API hides the complexity of the Bluetooth protocol stack, by exposing a simple set of Java API's.

The JSR-82 API provides you the options to do the following
1. Manage the Local Bluetooth Device settings.

2. Discover other Bluetooth devices in the neighborhood.

3. Search for Bluetooth devices on the discovered Bluetooth devices.

4. Connect to any of those Bluetooth service and communicate with it.

5. Register a Bluetooth service on the Local Bluetooth Device, so that other Bluetooth devices can connect to it.

6. Manage and control the communication connections.

7. Provide the security to all of the above options.

\section{PLATFORM}

\subsection{SYSTEM REQUIREMENTS}

\section{PC and SERVER}

Computer : IBM PC or $100 \%$ compatible

Ram : $256 \mathrm{MB}$

Operating System : Windows Xp (Service Pack 2) or newer

Disk Space

: $10 \mathrm{MB}+$ Bluecove package + JVM

Input device

: Standard Keyboard and Mouse.

Output device

: VGA and High Resolution Monitor.

Mobile Device

Java enabled mobile (CLDC-1.1 \& MIDP-2.0) with Bluetooth, capable of audio capturing, mixing and encoding=audio/amr.

\section{DESIGN OVERVIEW}

The Figure 2 shows the interface / model design. User has a Bluetooth enabled pc / laptop, Bluetooth enabled and java enabled mobile phone. User first have to ask login, if user successfully login then he can send to data to any registered member, whether the another user is online or offline it does not matter. Whenever another user comes online data is send to him if he is not in blacklist. First data or information is transferred from mobile to laptop or pc using Bluetooth then from laptop to centralized server where data is stored till it is not transferred to another user. Centralized server also stored details of user mean login information. Then if the user is not in the blacklist of another user it transforms to another user pc or laptop then on mobile using Bluetooth.

The UML deployment figure of our Implementation is shown in figure 3 .

\section{MODULES}

\section{Mobile Client Module}

Mobile Client is the client side module of the system. This module will allow client to connect to PC using Bluetooth and after connecting, client will send or receive files, messages, voice mail and also add or delete other clients from his/ her Blacklist. 


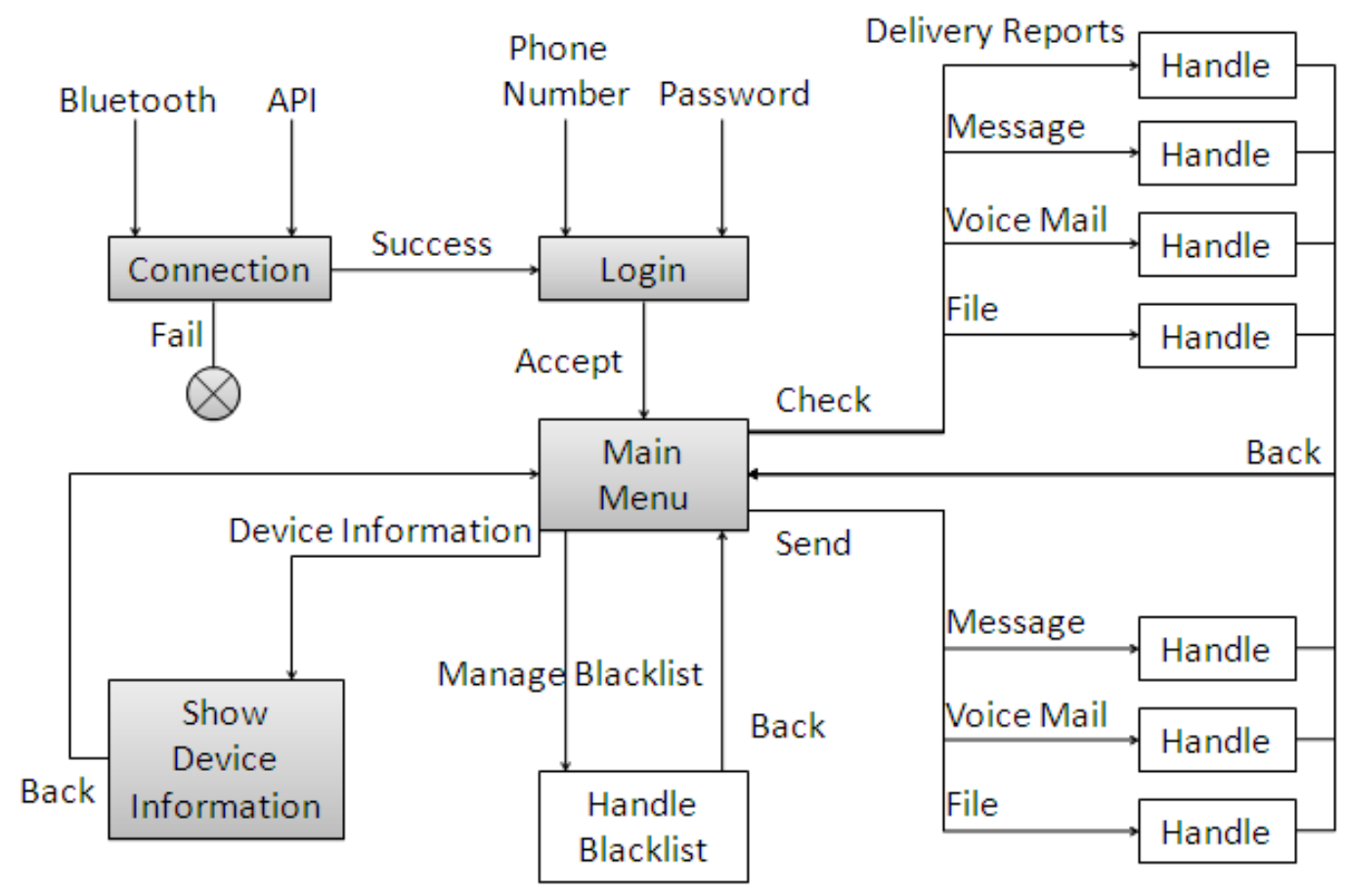

Figure 2 : Interface/Model Design

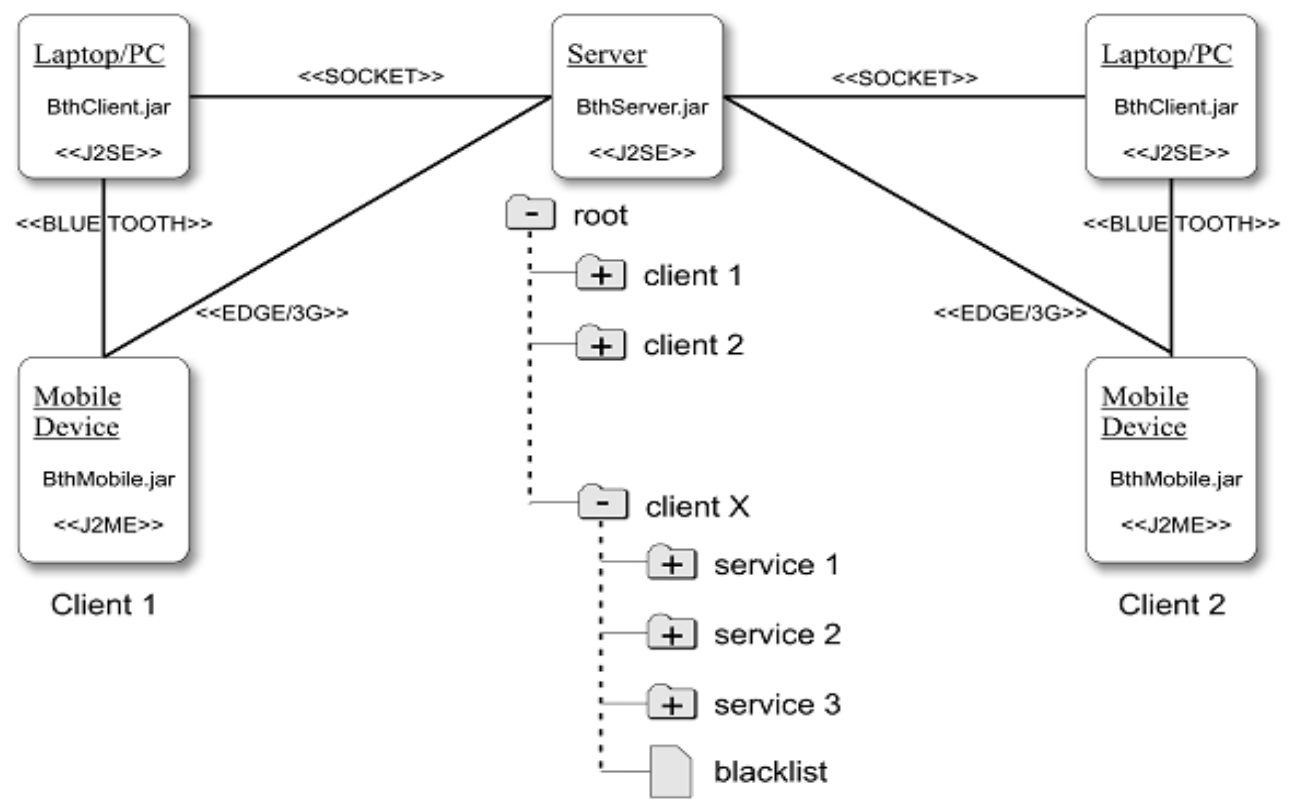

Figure 3: UML Deployment Diagram of Method 


\section{PC Module}

PC module is further divided into two modules: Client Module and Server Module.

\section{Server Module}

Server Module is the Server Side Module of the System. This Module will run on server and allow multiClients to connect to server. It acts as a medium to transfer data from one client to another client.

\section{Client Module}

Client Module is the Client Side Module of the System. This module will run on client PC and connect to server and also allow Mobile client to connect to Client.

\section{APPLICATION}

\section{- Educational Institutes}

Send a message/data to students/staff members. Generally, in rainy season it's difficult to convey message to students about classes etc. with the help of this application we can easily convey message to each registered student mobile.

- Data transfer between two persons in different cities Our this application makes easy to transfer data between two persons. Generally, problem regarding such transfer is that if the person is not available then he/she has to first copy it to his laptop/PC then send via e-mail or other modes of internet transfer and the receiver has to do the reverse. But with the help of this application, there is no need to copy data from mobile to laptop, no emails required, it will go directly as soon as user click send option.

\section{- Industries/companies}

In Industries/companies, the employees want to convey messages to other employees with this application easily.

\section{- Mobile E-learning}

Mobile E-learning is also a great application of our this system. There are other so many application of the developed system.

The Question arises why to use this application when so many Free message transfer softwares are available in the market.

\section{Reason for Using this application}

a. Based on new ideas and technology means using the concept of Bluetooth and open protocols.

b. Free services since no concept of GPRS (GPRS needed if not using laptop)

c. No need of service provider networks(like BSNL,TATA,AIRTEL) (Network service provider needed if not using laptop)

\section{CONCLUSION}

After the assessment of the developed system, is that although our project limited to basic services and security mechanism. Nevertheless we provide the most popular services and also retain the convenience and security of the design, and with continued effort it can become a useful part of future communication.

\section{ACKNOWLEDGMENTS}

Our thanks to IBM Research Lab, India for allowing us to develop such a model and then covert it in to an Application.

\section{REFERENCES}

[1] M. S. Gast, 802.11 Wireless Networks, First Edition, O'Reilly, 2002.

[2] D. Gratton, Bluetooth Profiles, The Definitive Guide, First Edition, Prentice Hall, 2003.

[3] B. Hopkings and R. Antony, Bluetooth for Java, First Edition, Apress, 2003.

[4] Kumar et. al., Bluetooth Application Programming with the Java APIs, First Edition, Morgan Kaufmann, 2004.

[5] J. Bray and C. Sturman, Bluetooth 1.1, Connect Without Cables, Second Edition, Prentice Hall, 2002.

[6] Bluetooth SIG, Specification of the Bluetooth System version 1.1, 2001.

[7] C. Gehrmann, Bluetooth Security White Paper,2 